\title{
Nô de Robert Lepage, ou Hiroshima mon amour revu par Feydeau
}

Marie-Chantal Killeen

University of Oxford (Lady Margaret Hall)

"Qu'est-ce que tu veux qu'on tourne à Hiroshima, sinon un film sur la Paix?"

—Duras $(1980,53)$

Dans son documentaire sur Hiroshima mon amour, Luc Lagier assimile le film-culte d'Alain Resnais à "un fantôme qui hante discrètement le cinéma contemporain" depuis 1959. ${ }^{1}$ Parmi la postérité protéiforme ${ }^{2}$ de ce revenant qui décidément n'en finit plus de revenir sur le grand écran, je me propose d'examiner ici le film Nô du cinéaste et dramaturge québécois Robert Lepage. Largement salué par le public et la presse au moment de sa sortie en 1998, Nô constitue un étonnant hommage au chef-d'œuvre de Resnais. Jusqu'à présent, la critique s’est contentée de mentionner au passage la référence intertextuelle sans pour autant montrer à quel point, et a fortiori comment et pourquoi, Nô s'imprègne d'Hiroshima mon amour. Car pour peu qu'on s'y attarde, on s'aperçoit que le film de Lepage est porté par un étourdissant jeu d'échos, de rappels, et de renvois à l'œuvre de Resnais qu'il réfléchit à la manière d'un de ces miroirs déformants que renferment les baraques foraines. Lepage n'y repère pas simplement un cadre exotique pour camper son histoire: il y puise au surplus tout un dispositif narratif qui lui permet de prendre en écharpe la question épineuse de l'Histoire et de la mémoire collective.

\section{Les Sept branches de la rivière Ota (1996)}

Signalons tout d'abord, en amont du film de Lepage, la pièce de théâtre Les Sept branches de la rivière Ota créée par sa troupe Ex Machina. ${ }^{3}$ Au départ, donc, Nô 
prend forme autour d'une de ces phrases incantatoires - "les sept branches de l'estuaire en delta de la rivière Ota" (Duras 1960, 34) - qui abondent dans le scénario de Marguerite Duras. Phrase que l'écrivaine avait elle-même dénichée chez John Hersey, l'un des tout premiers journalistes occidentaux à visiter Hiroshima au lendemain de la catastrophe pour y recueillir les témoignages de six hibakushas. ${ }^{4} \mathrm{~A}$ l'article de Hersey, paru en 1946 dans le New Yorker et qui a fait forte impression sur elle, Duras emprunte également la description étrangement inquiétante de la prolifération végétale dans les jours suivant le cataclysme, signe de "l’entêtement de la vie" (Duras 138) qui continue malgré tout, semblable en cela à la repousse des cheveux de la jeune tondue nivernaise. Visitant Hiroshima à l'occasion du cinquantième anniversaire du bombardement, Robert Lepage a été frappé lui aussi par la surprenante vivacité de la ville reconstruite (Fricker v).

Outre son titre, la formule durassienne prête aux Sept branches sa structure même. D'une durée de plus de sept heures, cette pièce-fleuve dans tous les sens du terme se compose de sept récits, conduits en parallèle sur trois continents. Traçant les grands bouleversements qui ont secoué le vingtième siècle - on y évoque les camps de concentration, les bombardements nucléaires, le SIDA -, les trames multiples de l'œuvre dramatique convergent au final sur la ville d'Hiroshima. Le cours d'eau de la rivière Ota, symbole premier chez Resnais de la coulée du temps qui emporte tout sur son passage, devient ainsi le site de confluence des péripéties et des personnages au dénouement.

Il reste que des sept volets de la saga, Lepage ne conserve pour son film qu'un seul, "Les mots," qui ressemble à première vue à ce que Montaigne qualifiait jadis de simple farcissure. Perçu au prisme d'Hiroshima mon amour, toutefois, cet intermède 
aux accents ludiques devient prétexte pour revenir sur ces événements d'octobre 1970 qui mirent fin à la Révolution tranquille.

\section{La Crise d'octobre au cinéma}

Dans l'histoire québécoise, la Crise d'octobre fait office de tournant. Selon le critique Gilles Marsolais, elle n'est rien de moins que "la clef indispensable à la compréhension du Québec contemporain et de ses aspirations” (Marsolais 20). Rappelons pour mémoire que les années 1960 furent ponctuées d'attentats à la bombe ciblant les hauts-lieux du pouvoir dans la province. ${ }^{5}$ Les auteurs de ces attentats militaient au sein du Front de libération du Québec (FLQ), mouvement nationaliste clandestin d'inspiration marxiste-léniniste, fondé en 1963 alors que les luttes de décolonisation battaient leur plein dans le monde entier. La violence atteignit son paroxysme au début du mois d'octobre 1970, avec les enlèvements de l'attaché commercial britannique, James Cross, puis du ministre provincial du Travail, Pierre Laporte, dont le corps sans vie fut retrouvé dans le coffre d'une automobile une semaine plus tard. L'intervention de l'État fut rapide et musclée. Pierre Elliot Trudeau, qui avait été élu premier ministre du Canada en avril 1968, proclama dans la nuit du 15 au 16 octobre la Loi sur les mesures de guerre. Quatre cent soixante-huit personnes, dont plusieurs journalistes et syndicalistes soupçonnés d'être partisans du FLQ, furent alors arrêtées sans mandat. Les images d'archives de la mobilisation des troupes quadrillant les rues de Montréal en chars d'assaut n'ont rien perdu de leur force percutante aujourd'hui.

Depuis lors, nombre de réalisateurs québécois se sont donné pour tâche de consigner ces événements dans la mémoire collective en les portant à l'écran. Caméra au poing, ils se sont exercés - et s'exercent encore - à dénoncer les abus de pouvoir 
de l'État et à défendre les revendications des felquistes, allant parfois jusqu'à légitimer leur recours à la violence. Le grand classique du genre, Les Ordres (1974) de Michel Brault (qui fut lui-même détenu pendant deux semaines), adopte l'esthétique du cinéma-vérité pour s'insurger contre la suppression des droits civiques durant la crise. Aux croisées du documentaire et de la fiction, son film s'inspire des témoignages d'une cinquantaine de Québécois qui avaient été arrêtés et incarcérés à l'époque, pour brosser le portrait composite de cinq victimes de l'arbitraire de la justice. Vingt ans plus tard, Pierre Falardeau revient à la charge avec son Octobre (1994). Scénarisé avec le concours de Francis Simard, l'un des membres de la cellule Chénier, ce réquisitoire sans appel contre le gouvernement envisage l'assassinat de Pierre Laporte du point de vue de ses ravisseurs. Plus récemment, le film Corbo (2014) de Mathieu Denis érige en martyr pour la cause souverainiste le jeune felquiste Jean Corbo, tué par la bombe qu'il posait à la Dominion Textile en 1966. En règle générale, ce cinéma engagé clame haut et fort la situation indigne d'un peuple colonisé, la fibre héroïque des militants et surtout l'échec tragique de leur combat pour l'indépendance du Québec (Marshall 37-45, Marsolais, Véronneau).

\section{Nô, ou la Crise d'octobre en comédie-bouffe}

Tourné en dix-sept jours en Super 16, l'une des pellicules préférées des cinéastes amateurs, Nô va prendre le contre-pied de ces récits fervents. Avec une verve pleine d'allant, le film de Lepage fait basculer le projet révolutionnaire dans une farce déjantée. Tout comme Marguerite Duras avait fait le pari audacieux de pallier l'impossibilité d'écrire sur l'événement irreprésentable d'Hiroshima en passant par une histoire d'amour tragique mais somme toute "racontable" (Duras 1980, 110), Lepage se propose d'imaginer la Crise d'octobre en immense quiproquo pour rendre 
compte de ce rendez-vous manqué du peuple québécois avec l'Histoire. À l'horreur innommable de la bombe atomique, Lepage juxtapose ainsi l'ineptie de révolutionnaires en herbe tout juste capables de faire sauter leur logis. Non pas que le cinéaste s'y fasse l'apologue du fédéralisme canadien, loin s'en faut, puisque le scepticisme joyeux de Nô n'épargne strictement personne. Peu importe que l'on soit souverainiste ou fédéraliste, chacun en prend ici pour son grade. Truffé d'extraits télévisés de l'époque - notamment des interviews avec Pierre Trudeau et Jean Chrétien (premier ministre à la sortie de $N o ̂$ ), et un vidéo-clip de Claude Ryan entonnant l'hymne national lors du vote référendaire -, le film se moque tout aussi allègrement des grandes figures politiques de la "campagne du Non."

Qu'on ne se méprenne pas: Lepage n'entend guère livrer de fines analyses sociologiques dans son film, pas plus qu'il ne cherche à donner des leçons, politiques ou autres. Le vœu d'en découdre avec les récits habituels naît plutôt de son désir de prendre du recul par rapport à ces années tumultueuses et d'en désamorcer la charge explosive par le rire. Il explique:

Nô était l'occasion de brosser une fresque d'époque avec un gars qui veut changer le monde en posant des bombes et une fille qui cherche à se réapproprier sa liberté. On commence seulement à être assez détachés de cette période pour pouvoir en parler avec humour. Car c'était une époque idéaliste mais aussi d'une maladresse incroyable. ${ }^{6}$

On sait que certains spectateurs du film de Resnais avaient jugé grossier, voire grotesque, le rapprochement de Nevers et d'Hiroshima. Pour Jean-Luc Godard, l'amoralité scandaleuse de l'œuvre émane de sa seule forme; ce qui ébranle derechef ce spectateur, c'est le nivellement des deux récits que pratique la caméra, montrant sans distinction aucune "l'amour ou l'horreur avec les mêmes gros plans" (Godard 
11). À propos de l'oxymore volontairement "sacrilège" (Duras 1980, 10) du titre, Marguerite Yourcenar avait riposté: “[C'est] comme si, après avoir été à Auschwitz, on écrivait Auschwitz, mon petit chou!" Le traitement irrévérencieux que Lepage fait subir aux déboires souverainistes a pareillement choqué plus d'un critique dans sa province natale. Certains, dont Robert Lévesque, n'y virent qu'une forme de désengagement potache. Il suffit de citer pour s'en convaincre le titre de sa recension cinglante: "Osaka, mon navet."

Si peu flatteur soit-il, le titre du compte-rendu de Lévesque souligne en toutes lettres le singulier hommage que le cinéaste québécois rend au film de Resnais. À vrai dire, tous les éléments qui font la force vive d'Hiroshima mon amour, depuis le croisement de l'Histoire collective et du drame personnel jusqu'à la mise en abyme comme levier critique de l'entreprise, en passant par la logique de substitution des personnages, vont se réunir dans Nô. Sauf qu'ici, mutatis mutandis, ils se travestissent tout de go en ressorts d'une comédie de boulevard dans laquelle vont s'empêtrer malgré eux les deux protagonistes. Nô se coule ainsi dans des formes connues, à savoir le film-culte et le théâtre dit "à formule," qu'il mine de l'intérieur en les faisant se chevaucher.

\section{L'enchevêtrement des récits}

Comme pour l'enchevêtrement des récits de Nevers et d'Hiroshima, une structure en contrepoint donne l'impulsion à la trame narrative de $N \hat{o}$. Mais au lieu de dépeindre, comme le font Duras et Resnais, une histoire au présent sans cesse happée et parasitée par les événements d'un passé qui menace à tout moment de resurgir, nous avons affaire à deux récits qui se déroulent concurremment le 15 et 16 octobre 1970 - soit en plein cœur de la crise -, l'un à Osaka et l'autre à Montréal. 
Bien que Sophie Maltais (Anne-Marie Cadieux) soit comédienne, à l'instar de l'héroïne d'Hiroshima mon amour interprétée par Emmanuelle Riva, la jeune Montréalaise est venue au Japon pour jouer non pas le rôle d'une infirmière dans un nouveau "film sur la Paix," mais celui d'une soubrette dans une pièce de Georges Feydeau, montée au Pavillon du Canada dans le cadre de l'Exposition universelle à Osaka. Pendant ce temps à Montréal, son ami Michel (Alexis Martin), révolutionnaire velléitaire et dramaturge en panne d'inspiration chronique, se laisse embarquer dans un complot terroriste par des amis venus se terrer chez lui pour échapper aux descentes des gendarmes.

Faisant alterner les plans en couleurs pour les séquences au Japon, et en noir et blanc pour les séquences au Québec - clin d'œil éventuel au cinéma-vérité de Michel Brault qui en faisait de même dans Les Ordres -, Nô se place d'emblée sous le sceau du double. À commencer bien sûr par le jeu de mots du titre, qui renvoie aussi bien au théâtre japonais traditionnel mis en scène à l'amorce du film, qu'à l'échec du référendum de 1980 sur l'indépendance du Québec par lequel il s'achève. À la différence d'Hiroshima mon amour qui s'ouvre sur les plans serrés de corps nus enlacés, recouverts tour à tour des cendres atomiques puis des 'sueurs de l'amour accompli” (Duras 1980, 10) - figure emblématique d'une œuvre qui s'attache à conjuguer la mort et l'érotisme -, Nô présente l'insert de deux réveille-matin, placés côte à côte dans l'appartement de Michel, l'un affichant l'heure locale et l'autre celle d'Osaka, auxquels correspondent les deux horloges de voyage emportés par Sophie au Japon. Façon de prévenir gentiment le spectateur qu'il ne sera guère question dans ce film de mort et d'amour ineffables, mais tout au plus d'une mécanique divertissante, composée d'aventures grivoises et d'actes de sabotage ratés. 
L'image des deux cadrans donne le coup d'envoi aux mésaventures rocambolesques de Sophie et de Michel, qui tout au long du film peineront à remettre, comme l'expression le veut, les pendules à l'heure. Source permanente de confusion, le décalage horaire déclenche une véritable cascade d'imbroglios et de quiproquos qui feront déraper et s'emmêler leurs deux histoires. C'est ainsi que Sophie, désemparée d'apprendre qu'elle est enceinte sans savoir de qui, se fourvoie dans ses calculs de fuseaux horaires en contactant Michel du Japon. Elle l'appelle donc en pleine nuit alors que des copains, sympathisants du FLQ, arrivent au débotté pour demander asile chez lui. Au bruit qu'elle perçoit à l'autre bout du fil, elle déduit que Michel la trompe - “à quatre heures du matin, c'est sûrement pas les témoins de Jéhovah," lui dit-on d'un air entendu - et décide de lui rendre la pareille. Moyennant quoi Sophie, à l'image de la Française d'Hiroshima, finira sa dernière nuit au Japon dans les bras d'un inconnu, dans ce cas-ci Walter (Richard Fréchette), l'attaché culturel de l'ambassade du Canada à Tokyo que l'un des acteurs de la troupe qualifie, en bon québécois, d'“hostie de bourgeois, d'opportuniste, d'insignifiant [et] de fédéraliste fini.” (Pas la peine de préciser qu'il manque à Walter le charisme certain de l'amant japonais joué par Eiji Okada dans Hiroshima.) N'empêche que ce premier malentendu paraît bien mince comparé à l'énorme bévue de Michel, qui par mégarde donne à ses amis le réveille-matin réglé à l'heure japonaise pour détoner leur bombe, et du coup fait exploser son propre appartement.

À déplier de la sorte ces malentendus et les péripéties alambiquées qu'ils déchaînent, on pourrait se croire à mille lieues de l'univers imaginé par Resnais et Duras. Mais à y regarder de plus près, force est de reconnaître que la méprise constitue aussi l'un des ressorts essentiels d'Hiroshima mon amour. Ce film repose à la base sur une série d'équivoques, dont la plus importante à coup sûr est le saut 
dialectique qui s'opère entre le mélo sentimental et le récit impossible sur Hiroshima. Osant "[mettre] face au chiffre énorme des morts d'Hiroshima l'histoire de la mort d'un seul amour inventé par [Duras]" (Duras 1960), le film de Resnais tire sa vitalité et son efficace d'un mouvement de va-et-vient incessant entre Hiroshima et Nevers, c'est-à-dire entre l'Histoire "avec sa grande hache," comme disait Georges Perec, et une toute petite "histoire de quatre sous" (Duras 1980, 118). ${ }^{7}$ Sans ce glissement à double sens qui s'effectue entre le collectif et le personnel, l'œuvre serait délestée de sa dimension allégorique et de sa portée politique tout à la fois.

Renchérissant sur Resnais, Lepage va amplifier et détourner ce mouvement jusqu'à la caricature. Le critique Christopher E. Gittings a bien montré comment action politique et libertinage se confondent systématiquement dans $N \hat{o}{ }^{8}$ On a beau vouloir s'impliquer et faire son entrée dans la sphère publique, les gestes que l'on pose sont non seulement mal interprétés par autrui, mais ravalés chaque fois à une banale histoire de sexe. D'un bout à l'autre du film, les allusions aux liaisons amoureuses viennent constamment redoubler, en les brouillant, les références au combat politique. J'en veux pour preuve la comparaison, peu subtile mais non moins efficace, que dessine $N o ̂$ entre l'héroïne enceinte qui souhaite disposer librement de son corps ${ }^{9}$ et le peuple québécois appelé à forger son propre destin. Filant lourdement cette analogie, Nô fait même coïncider la fausse-couche de Sophie et le moment où avorte la révolte felquiste. (Parallèle mis en valeur, ainsi que l'observe Peter Dickinson, par le passage définitif du noir et blanc à la couleur qu'opère un plan serré sur la mini-jupe blanche et les cuisses de Sophie, le long desquelles dégoulinent des filets de sang [Dickinson 148-9].) Le dénouement du film revient avec insistance sur ce curieux tandem que forment l'individu et le corps politique. Nous retrouvons, dix ans plus tard, Sophie et Michel qui regardent à la télévision les résultats du 
référendum sur la souveraineté. Leur discussion à propos de la défaite politique a tôt fait de se muer en une conversation badine au cours de laquelle Michel cherche à convaincre Sophie de compenser l'apparente stérilité du projet collectif par la conception d'un enfant. Qu'est-ce à dire, sinon qu'en cette nouvelle ère individualiste où s'étiolent les grands récits, les aspirations politiques se rétrécissent comme peau de chagrin pour se reporter sur la famille nucléaire? Il n'est certainement pas anodin qu'à la fin d'Hiroshima mon amour, suivant une espèce de baptême réciproque, les protagonistes anonymes se donnent pour noms "Nevers-en-France" et "Hiroshima." L'épilogue de $N \hat{o}$, en revanche, retrace dans le sens inverse ce passage allégorique du couple à la nation.

\section{Transfert et quiproquos}

Autre point de convergence important des deux œuvres: la logique de permutation qui gouverne les rapports entre personnages. La dramaturgie durassienne du désir, tout entière soumise aux mécanismes du transfert et de la délégation, témoigne d'une profonde porosité des personnages. Le Japonais cultive à dessein cette ambiguïté, persuadé que le souvenir enfoui de Nevers est le sésame qui lui fera pénétrer dans l'intimité secrète de la Française. Il interroge l'actrice sur son passé, lui donne à boire, et même usurpe la voix de l'amant allemand abattu dont il parviendra petit à petit à s'arroger la place.

Cheville ouvrière de l'intrigue d'Hiroshima mon amour, la confusion des

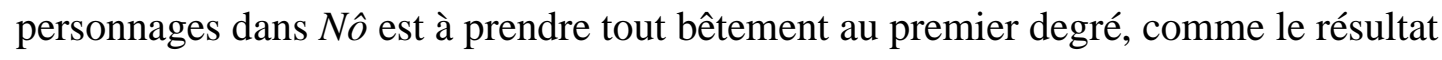
de quiproquos et d'erreurs d'identité burlesques dans des scénarios de galipettes et de cocufiage droit sortis du théâtre de Feydeau. Au moteur secret de la cure psychanalytique, Lepage substitue gaillardement les conventions les plus éculées du 
théâtre de boulevard. Témoin la séquence qui regroupe Sophie et Walter dans la chambre d'hôtel de celle-ci. Vient d'abord frapper à la porte Patricia (Marie Gignac), qui surprend son époux en flagrant délit (lequel s'exclame "Ma femme!," comme il se doit, en courant se cacher dans le placard). Arrive ensuite François-Xavier (Éric Bernier), le soupirant éconduit de Sophie dont celle-ci n'arrive pas à se débarrasser. Valises en main, elle tente de s'enfuir mais voilà que la chambre s'avère être sans issue: la porte ne donne plus sur le couloir de l'hôtel mais sur le décor en carton-pâte de la Dame de chez Maxim. L'héroïne interloquée se trouve ainsi bouche bée sur la scène de théâtre, flanquée de comédiens qui saluent l'auditoire au baisser du rideau. Le fondu enchaîné sonore qui incorpore la musique japonaise du nô aux applaudissements des spectateurs ne fait qu'accentuer l'étrangeté et le comique de la scène.

Aussi la tekhnè dans Nô vient-elle suppléer à la (pseudo)-séance psychanalytique que le Japonais met patiemment en scène dans Hiroshima mon amour. C'est une cabine Photomaton à l'Exposition universelle qui tient lieu de support aux transformations des personnages et des situations. Comme par magie, elle dévoile en un instant fulgurant la psyché de ceux qui viennent s'y faire une photo de “passeport” (c'est-à-dire le laissez-passer pour l'Exposition) en donnant à voir très concrètement les fantasmes et les peurs qui les habitent. La cabine se transmue pour le coup en une espèce de confessionnal kitsch qui n'est pas sans faire penser au premier film de Lepage. ${ }^{10}$

Parmi les personnages qui défilent dans le Photomaton, il faut accorder une attention particulière à Hanako (Marie Brassard), que le largage de Little Boy sur Hiroshima a rendue aveugle. Quand elle retire ses verres fumés pour la photo, le flash de l'appareil fait réapparaître tout d'un coup celui de la déflagration atomique. De 
simple objet de pacotille pour touristes, la "photo-passeport" de l'Expo ranime ici le plus angoissant des souvenirs de la Japonaise. Peut-être objectera-t-on que cet enchaînement verse dans la facilité et la complaisance, pour ne pas dire le mauvais goût. Il faut admettre que ces images du champignon nucléaire - vestiges du projet beaucoup plus sobre que Lepage menait dans les Sept branches de la rivière Otatombent plutôt à plat dans le cadre désinvolte de $N \hat{o}$, si bien qu'elles n'apparaissent plus que comme matière à recycler, ou pis encore à esthétiser. Mais il y a fort à parier que l'intercalation de ces images n'est pas tout à fait gratuite. Duras n'envisage-t-elle pas dans son scénario la surimpression du crâne brûlé d'un hibakusha et de la Place de la Paix (Duras 1980, 28)? Dans Les Sept branches de la rivière Ota, en tout cas, Lepage semble prendre acte de la question délicate de la représentation du désastre et des effets spectaculaires qu'elle déploie. Tournant un documentaire pour commémorer le cinquantième anniversaire d'Hiroshima, le personnage de Patricia pousse l'inconvenance jusqu'à proposer le fondu enchaîné du crâne d'une rescapée de Theresienstadt avec le fameux Dôme du Palais de l'Industrie, conservé en l'état depuis le bombardement atomique. Quid, désormais, de cet interdit de la représentation d'Hiroshima qui pesait encore sur la démarche de Resnais et de Duras, et qui fut longtemps tenu pour gage de fidélité à l'incommensurabilité de l'événement?

\section{Esthétique et politique}

Que la forme engage le fond, c'est ce que soutiennent Jean-Luc Godard et Jacques Rivette dans leurs analyses des travellings et des gros plans d'Hiroshima mon amour. Pour eux, il va sans dire que le montage et les prises de vue sont porteurs d'une idéologie. Il est vrai, en ce qui concerne Resnais, que plus son film avance, et plus la 
caméra épouse les mouvements et les soubresauts de la conscience de l'héroïne, comme pour mieux nous faire partager sa détresse et son dépaysement. Par contraste, la perspective dominante dans l'œuvre de Lepage - si tant est qu'on puisse même affirmer que la caméra privilégie un certain point de vue, ce qui est loin d'être sûr -, reste difficilement cernable. Le regard espiègle que $N o ̂$ pose sur le monde tiendrait davantage d'une sorte d'impersonnalité flaubertienne, teintée d'une sempiternelle ironie, placée bien en retrait des événements qu'elle détaille. On peine à identifier clairement dans l'œuvre quelque chose qui serait comme un "message.” De là sans doute la divergence notable des réactions que le film a suscitées, interprété tantôt comme souverainiste, tantôt comme anti-souverainiste.

À cela s'ajoute le fait que $N o ̂$ se présente bien souvent comme le versant négatif (au sens photographique du terme) du film de Resnais dont il inverse les valeurs. Le recours aux fondus enchaînés dans Hiroshima mon amour a pour effet de suturer les ellipses temporelles et de télescoper les personnages. Lepage, de son côté, trafique d'ingénieuses coupes et collures de plans qui “citent” ceux de Resnais tout en resserrant, par la même occasion, les liens entre les divers récits, lieux, et personnages de $N$ ô. C'est ainsi, par exemple, que la caméra passe quasi imperceptiblement de François-Xavier allongé sur son lit d'hôtel, à Walter, étendu sur le lit de Sophie dans la même pose. Non seulement ces plans de raccord nous invitent à dresser un parallèle entre les prétendants ridicules de l'héroïne, ils ont en outre le mérite de se greffer, en se superposant pour ainsi dire, au célèbre fondu enchaîné d'Hiroshima mon amour, au moyen duquel l'amant japonais endormi se transforme subitement en soldat allemand, gisant sur le quai au bord de la Loire. Le comédien amouraché de Sophie et son amant d'un soir se font ainsi les doubles absurdes des amants de la Française. 


\section{Mises en abyme}

Mais plus encore que le dispositif d'entrecroisement des récits, le glissement du collectif au personnel, la substitution des personnages et le travail sur l'image et le son, c'est assurément l'aspect autoréflexif du film de Resnais qui laisse la marque la plus durable sur l'œuvre de Lepage. On se souvient que Hiroshima mon amour soumet sa propre démarche à l'examen critique en pointant, par d'habiles mises en abyme, les lacunes et les écueils que présentent d'autres approches que la sienne. Toute la première partie du film s'attelle à refuser le genre documentaire et à problématiser l'image d'archive. Dans des travellings le long des couloirs de l'hôpital et des plans fixes des escaliers et des passerelles vides du musée, la caméra investit ces espaces muets afin de souligner l'inadéquation d'une mémoire qui se voudrait institutionnelle, c'est-à-dire froide et objective. Tant qu'elle ne passe pas par l'expérience et qu'elle ne parle pas directement au corps, celle-ci laisse "la foule indifférente" (Duras 1980, 64) sur la Place de la Paix. Quant aux tentatives d'appréhender le désastre par le biais de la fiction historique, le problème reste entier. Si réalistes soient-elles, les reconstitutions s'exposent au risque de banaliser l'horreur qu'elles décrivent. Il n'est pas jusqu'à l'image tétanisante du champignon nucléaire, que Resnais décide du reste de supprimer du scénario durassien, qui ne s'use à force d'être vue et revue. C'est là le saisissant paradoxe auquel Hiroshima mon amour ne cesse de nous confronter: la répétition consigne à l'oubli. À telle enseigne que plus "personne ne regarde" les plaques, les mémoriaux, et même les figurants grimés en hibakushas dans les rues d'Hiroshima: “on a l'habitude à Hiroshima de voir tourner des films sur Hiroshima" (Duras 1980, 64), explique l'actrice française. À quoi elle ajoute, sans grande conviction, "Il y a bien des films publicitaires pour le savon. Alors... à force... peut-être" (65). Tourner un film sur Hiroshima reviendrait à 
emprunter les armes du capitalisme pour vendre ni plus ni moins l'idée de la Paix. Le commentaire de la Française nous conduit par ailleurs à sonder l'ambiguïté que recèle cette vaste entreprise de commémoration. Resnais nous en met plein la vue dans Hiroshima mon amour de ce qu'il conviendrait de taxer de tourisme de l'horreur. ${ }^{11}$ Sur fond d'une musique de cirque enjouée, un flot d'images évoque éloquemment la conversion indécente de la catastrophe en spectacle et en source de revenus.

Lepage pour sa part use du procédé de la mise en abyme pour nous faire réfléchir à la fois sur ce que l'on pourrait appeler la mémoire “officielle” et sur la marchandisation du patrimoine culturel. L'Exposition universelle fait figure de pendant spéculaire du musée d'Hiroshima, en ce sens qu'elle est chargée elle aussi de fabriquer pour consommation étrangère des récits officiels sur la nation. Ou pour le dire plus pompeusement, en citant la langue de bois du correspondant de RadioCanada: "cet événement international aura permis aux peuples de la Terre de diffuser la quintessence de leur culture respective dans une atmosphère de fraternité remarquable." De cette folklorisation de la culture résulte un véritable bêtisier de clichés que Lepage se donne à cœur joie de décliner, notamment dans de faux reportages où défilent des Japonais déguisés en gendarmes de la Police Montée et dans une vidéo promotionnelle qui s'évertue à attirer des touristes au Québec en leur vantant le charme inouï des cabanes à sucre et du Bonhomme Carnaval.

Lepage choisit pour opérateurs de son analyse deux pièces de théâtre qui semblent à première vue n'avoir rien en commun, hormis le fait que la première est présentée au Pavillon du Japon et la seconde au Pavillon du Canada. En hommage aux cultures asiatiques qui de tout temps le passionnent, ${ }^{12}$ Lepage place d'abord en abyme, dans l'une des séquences inaugurales du film, l'extrait d'un spectacle nô. Cette pantomime stylisée, dont la gestuelle dépouillée évoque par certains côtés le jeu 
distancié et la diction monocorde des acteurs d'Hiroshima mon amour, est enchâssée dans un de ces faux reportages de Radio-Canada dont Lepage fait son miel. Le voiceover du journaliste nous en donne un synopsis dans sa chronique. L'acteur nô, explique-t-il, "revêt les traits d'une jeune femme exilée qui, après avoir tenté de noyer ses tourments dans un torrent impétueux, retrouve sa route grâce aux lueurs d'un brasier allumé en lettres de feu par son amant guerrier." Voilà qui pourrait condenser (avec un lyrisme fort appuyé, on en convient ${ }^{13}$ ) l'essentiel de l'action d'Hiroshima mon amour et de Nô. Loin de leur terre natale, la Française et la Québécoise traversent toutes deux une crise qu'elles s'emploient à noyer dans l'alcool et l'adultère. De même que la Française réussit à se délivrer du souvenir traumatique du soldat défunt par le biais du Japonais, de même Sophie finit par renouer avec Michel, ce "samouraï" pour le moins picaresque.

Si la mise en scène du théâtre nô vise surtout à creuser l'écart comique entre le modèle noble aux allures épiques et sa copie dégradée ${ }^{14}$ en multipliant les jeux de miroir et les coups de coude complices, la référence à La Dame de chez Maxim de Feydeau sert davantage à formuler la critique de Nô. Car le projet insensé de représenter le Québec à l'Exposition universelle de 1970 par un vaudeville français de 1899 est le symptôme d'une affligeante schizophrénie culturelle. (On ne manquera pas de relever l'allusion ironique à Lepage lui-même, metteur en scène expérimental qui se lance ici dans la comédie de boulevard ${ }^{15}$.) Rien ne cristallise mieux cette dissonance cognitive que le cri de "Vive la France!" qui marque le dénouement de la pièce de Feydeau, et qui bien sûr fait résonner en creux le "Vive le Québec libre!" qu'avait lancé le président de Gaulle - lors de l'Exposition universelle à Montréal, justement - trois ans plus tôt. L'incongruité d'un tel choix artistique ne saurait passer 
inaperçue. Au cours d'un dîner copieusement arrosé de saké, Sophie se déchaîne contre Walter et son épouse Patricia:

À part de ça, là, vous êtes diplomate, vous? Vous allez m'expliquer ça, comment ça se fait qu'on fait venir un metteur en scène français à Montréal, pour nous apprendre à parler avec un accent français, dans une mauvaise pièce française, pis que c'est ça qui représente le Canada à l'Exposition universelle d'Osaka? Ben, je vais vous le dire pourquoi, moi. Parce qu'on est un peuple de colonisés!

L'accent parisien que feint la très snob et pimbêche Patricia ne fait qu'apporter une preuve supplémentaire au diagnostic de l'héroïne. Cette séquence gagne à être rapprochée de celle qui se déroule dans la discothèque du Pavillon du Québec où s'esquive Sophie (telle la Française d'Hiroshima qui s'était réfugiée dans la boîte de nuit Casablanca). On y joue un tube québécois de 1969, “Tout écartillé” de Robert Charlebois. Émaillée de savoureux québécismes et rappelant à point nommé qu'“Oh non, Paris c'est pas mon étable," la chanson québécoise vient faire contrepoids à la comédie de boulevard.

Dans cette opposition du théâtre de Feydeau et de la "parlure" québécoise, c'est évidemment tout le rapport à la langue, question névralgique s'il en est, qui entre en jeu. (Encore que La Dame de chez Maxim faisait déjà de la langue un de ses thèmes privilégiés. ${ }^{16}$ ) Comme l'indique d'emblée "Les mots," le titre du volet des Sept branches qui contient en germe le récit de $N \hat{o}$, la langue représente le nerf de la guerre dans la lutte pour l'indépendance. Sensible au pouvoir des mots, Michel insiste pour corriger le communiqué bourré de fautes de français que ses camarades ont rédigé à chaud. Michel leur reproche en somme d'employer une langue carencée à l'image du peuple dépossédé qu'ils prétendent émanciper: 
C'est pas toi qui disais que notre premier combat, c'est la langue?

Notre première forme de résistance, c'est la langue. La façon dont on la parle, les mots qu'on utilise, les mots qui ne sont pas les mêmes que ceux de l'oppresseur! Une volonté de ... transcender une langue aliénée, misérable pour accéder à une langue adulte, souveraine! ... Puis maintenant qu'il faut l'utiliser, la langue, tu nies le pouvoir des mots! Par le truchement d'Hanako, l'interprète japonaise et hibakusha frappée de cécité, Nô touche également la question de la traduction qui fera décupler les méprises, culturelles et linguistiques cette fois. C'est Hanako qui, prononçant le fameux dicton Traduttore tradittore, rappelle que le traducteur doit veiller en permanence à ce que des erreurs ou des étourderies ne détournent subrepticement le sens d'un propos. Fautil y voir une allusion au défi que pose le bilinguisme canadien? Une manière d'avertissement au sujet de la distorsion de l'idéal politique du moment qu'on s'acharne à lui donner une forme concrète? À moins que ce ne soit à nouveau une allusion autoréflexive à $N o ̂$ et à son adaptation "traîtresse" du film de Resnais?

\section{Conclusion}

En exploitant un genre populaire dévalorisé, Hiroshima mon amour et Nô abordent tous deux en oblique des événements dont la représentation frôlerait le tabou. Mais tandis que le mélodrame constitue chez Duras et Resnais un puissant vecteur à l'affect, la comédie bouffonne de Lepage fait table rase de ce pathos, qu'elle dégonfle et même évacue sous l'effet du rire. Au bout du compte, comme le note Matthew Evangelista, Nô ressemblerait moins au théâtre dont il porte le nom qu'à un kyōgen, l'interlude ludique que renferment les pièces nô classiques. C'est que l'œuvre de Lepage n'offre, en définitive, aucune prise au sentiment. Y sont tournés en dérision 
des sujets tels le combat politique, les attentats à la bombe, ou la grossesse non désirée, qui partout ailleurs forceraient le sérieux et l'émotion. Tout se joue dans le ton (ton qui détonne encore plus de nos jours, alors que se multiplient les attentats terroristes): à la mélancolie envahissante d'Hiroshima mon amour et à l'héroïsme tragique qui est le fond de commerce du cinéma sur la Crise d'octobre, Lepage oppose non sans hardiesse l'apparente légèreté du vaudeville. C'est dans l'intervalle d'avec le modèle de Resnais, comme dans la déviation par rapport aux discours hégémoniques sur octobre 1970, que Nô acquiert son sens et son intérêt.

Pour Lepage, le théâtre de Feydeau est tout ensemble la cible et l'instrument de sa critique. Si la comédie de boulevard se fait l'indice patent d'une colonisation culturelle dont les Québécois auraient longuement souffert, il reste que c'est en maniant les outils de la farce que Lepage tente de mettre à nu cette impasse qui caractérise à ses yeux les débats politiques de l'époque. Impression d'impuissance que les personnages de $N \hat{o}$, en proie à d'infinis atermoiements, illustrent parfaitement. Quand bien même on souhaiterait changer la situation politique, le discours engagé semble destiné à tomber dans l'oreille d'un sourd. Quoique Sophie veuille se consacrer sérieusement au théâtre et Michel défendre la cause qui lui tient à cœur, l'une doit se résoudre à jouer dans une pièce de boulevard surannée tandis que l'autre, terrassé par l'angoisse de la page blanche, se découvre dans la peau d'un révolutionnaire raté.

Là encore, on pourrait penser que Nô reste traîtreusement fidèle à Hiroshima mon amour. L'œuvre de Resnais et de Duras ne cesse de tourner autour de ces événements qui nous dépassent sur le vif, ces expériences que l'on n'est pas à même de saisir, et encore moins de maîtriser, au moment où on les vit. Tout se passe comme si, à Hiroshima, la Française reconnaissait s’être trompée en quelque sorte de scénario. 
“[D]avantage que les autres femmes 'amoureuse de l'amour même”" (Duras 1980, 154), elle s'était imaginé vivre à Nevers une histoire d'amour légendaire à nulle autre pareille. À travers sa rencontre avec le Japonais, elle se rend enfin à l'évidence qu'on ne meurt pas d'amour. On pourrait en dire autant du déroutement des personnages de Nô qui ne savent plus quelle réplique donner: s'étant pris à tort pour des acteurs dans un drame, ils se retrouvent plantés, à leur corps défendant, dans un décor de vaudeville.

\section{Notes}

\section{Ouvrages cités}

Brault, Michel. Les Ordres. Les Productions Prisma Films, 1974, 107 minutes.

Charest, Rémy. Robert Lepage: Quelques zones de liberté. Québec: L'instant même, 1995.

Coulombe, Michel. "Entretien avec Robert Lepage. Propos recueillis par Michel Coulombe." Nô. Scénario du film de Robert Lepage. Laval: Les 400 coups, 1998. 95100 .

Denis, Mathieu. Corbo. Max Films Productions, 2014, 119 min.

Dickinson, Peter. Screening Gender, Framing Genre. Canadian literature into film. Toronto: Toronto University Press, 2007.

Domarchi, Jean, Jacques Doniol-Valcroze, Jean-Luc Godard, Pierre Kast, Jacques Rivette et Eric Rohmer. "Hiroshima, notre amour. Table ronde." Cahiers du cinéma XVII. 97 (juillet 1959): 1-18.

Dunđerović, Aleksandar. The Cinema of Robert Lepage: The Poetics of Memory. London: Wallflower Press, 2003.

Duras, Marguerite. Hiroshima mon amour. Scénario et dialogue. Paris: Gallimard, 1960.

—. "Je me souviens." Les yeux verts. Cahiers du cinéma, 312-313 (juin 1980): 34.

Evangelista, Matthew. "Québec: oui, no, or femme." Gender, Nationalism and War. Conflict on the Movie Screen. Cambridge: Cambridge University Press, 2011. 203252. 
Falardeau, Pierre. Octobre. Office National du Film, 1994, 100 minutes.

Fricker, Karen. "Introduction." The Seven Streams of the River Ota. Methuen: York, 1996. v-vii.

Gittings, Christopher E.. Canadian National Cinema: Ideology, difference and representation. London / New York: Routledge, 2002.

Harvie, Jennifer. "Transnationalism, Orientalism, Cultural Tourism: La Trilogie des dragons and The Seven Streams of the River Ota." Theater [sic] sans frontières.

Essays on the Dramatic Universe of Robert Lepage. Joseph I. Donohoe Jr. et Jane M. Koustas (dir.). East Lansing: Michigan State University Press, 2000, 109-25.

Hersey, John. "Hiroshima.” The New Yorker. août 1946. Réimprimé dans Hiroshima. Londres: Penguin Books, 1946 [2001].

Lagier, Luc. "Hiroshima le temps d'un retour." documentaire, Arte, 2004, 23 minutes.

Lepage, Robert. Le confessionnal. Alliance VivaFilms, 1995, 100 minutes.

—. The Seven Streams of the River Ota. Methuen, York, 1996.

—. Nô. Communications Co., In Extremis Images, 1998, 85 minutes.

—. Cité sur le site L'Indépendance du Québec. URL: http://www.independancequebec.com/flq/octobre/film_no.html. Page consultée le 4 février 2016.

Lévesque, Robert. “'Osaka, mon navet.' Ouvrage recensé: Nô de Robert Lepage.” 24 images 93-94 (1998): 88-89.

Marshall, Bill. Québec National Cinema. Montreal, Kingston, London, Ithaca: McGill-Queen's University Press, 2001.

Marsolais, Gilles. "Octobre au cinéma: la mouvance révélatrice d'une démocratie en péril.” 24 Images 93-94 (1998 ): 20-22.

Resnais, Alain. Hiroshima mon amour. Pathé Overseas Productions, 1959, 90 minutes.

Véronneau, Pierre. "Les événements d'octobre au cinéma.” Québec Studies 11 (19901991): 29-36.

Yourcenar, Marguerite. "Entretien avec Michèle Stouvenot." Le Journal du dimanche, le 2 décembre 1984. 
${ }^{1}$ Parfois même ce revenant s'affiche de façon très nette, comme dans le film H. Story (2001) du réalisateur japonais Nobuhiro Suwa. Racontant le tournage raté d'un remake d'Hiroshima mon amour, Suwa semble y prendre au pied de la lettre l'injonction de Marguerite Duras, à savoir qu'il est "[i]mpossible de parler de HIROSHIMA," et que par conséquent, "[t]out ce qu'on peut faire c'est de parler de l'impossibilité de parler de HIROSHIMA" (Duras 1980, $10)$.

${ }^{2}$ Sur ce genre de 1" "hiroshimade" que fondent Resnais et Duras, voir aussi mon article "Tout vu, rien inventé: Hiroshima mon amour dans l'œuvre de Chloé Delaume," à paraître.

${ }^{3}$ Pour une discussion de la genèse de la pièce et du film, voir Dunđerović (95-104).

${ }^{4} \mathrm{C}$ 'est ainsi que l'on désigne au Japon les survivants de la bombe atomique.

${ }^{5}$ Parmi lesquels le centre de recrutement pour l'armée canadienne à Sherbrooke, la caserne du régiment des Black Watch à Montréal, la Bourse de Montréal, l'université McGill, le grand magasin Eaton, et le monument de la reine Victoria à Québec.

${ }^{6}$ À ces propos de Lepage cités sur le site Indépendance du Québec, on pourrait ajouter ses remarques tirées de son entretien avec Michel Coulombe. Lepage y insiste sur le fait qu'il ne se moque pas des motivations politiques des combattants: "ces gens-là étaient inspirés et il se passait là quelque chose d'authentique. Mais il s'agissait tout de même de professeurs de philosophie qui allaient poser des bombes. Alors pour ce qui est de l'efficacité..." (Coulombe 97).

${ }^{7}$ Gardons-nous cependant de conclure que cette homologie pose une équivalence entre le trauma de Nevers et celui d'Hiroshima. De toute évidence, le réalisateur et la scénariste mettent en rapport ces deux expériences en apparence incommunicables pour faire rejaillir le sentiment de dépossession et d'incompréhension qui leur est commun. Nevers passe pour un reflet nécessairement déformé et partial de la grande Histoire, qui elle aussi échappe à l'entendement de celui qui la subit. Il s'agit donc, dans un cas comme dans l'autre, de révéler l'échec de la représentation. C'est justement cet échec qui donnerait une caution éthique au projet de Duras et de Resnais. 
${ }^{8}$ C'est ce que fait Sophie, qui tire des conclusions hâtives quand elle appelle Michel du Japon, et la concierge à Montréal qui surprend deux hommes dans l'appartement qu'elle fait visiter à deux éventuels locataires. Elle suppose qu'il s'agit d'un rendez-vous érotique alors qu'en fait ce sont des policiers planqués en surveillance en face de chez Michel. (Parce que les forces de l'ordre, est-il besoin de le noter, s'avèrent tout aussi incompétentes que les militants dans $N o \hat{o}$.) ${ }^{9}$ Bien que l'avortement ait été décriminalisé au Canada en 1969, la loi stipulait encore qu'une femme ne pouvait se faire avorter que si un comité composé de trois médecins estimait que la vie de la femme était en danger. D'où l'intention de Sophie, au début du film, de reporter son départ du Japon afin d'interrompre sa grossesse en toute sécurité.

${ }^{10}$ Le Confessionnal (1995) s'est construit lui aussi en dialogue constant avec un film classique: I Confess (1953) d'Alfred Hitchcock.

${ }^{11}$ Il faut songer à ces images d'une guide touristique souriante, tenant son microphone dans le car "Atomic Tours"; aux étagères de l'Hiroshima Gift Shop chargées de maquettes décoratives du Palais de l'Industrie et d'autres bibelots dérisoires; à la bannière d'une boutique de souvenirs interpellant les visiteurs: "How aboute a card for correspondense commemorating today's occassion?" [sic].

${ }^{12}$ La Trilogie des dragons, Le Dragon bleu, et Eonnegata disent assez la prédilection de Lepage pour les cultures japonaise et chinoise, dont il parle dans un entretien avec Rémy Charest (Charest 47-55). La critique Jennifer Harvie le prend cependant à parti sur cette question en l'accusant d'“exotisme récidiviste” (Harvie 123). Selon elle, les pays de l'Extrême Orient, insuffisamment différenciés et fortement fétichisés dans l'œuvre de Lepage, ne seraient plus qu'un support fantasmatique.

${ }^{13}$ Rapprocher le "brasier allumé en lettres de feu" de la bombe qui réduit l'appartement de Michel en décombres exige certes un effort d'imagination. On aura compris que même les personnages qui ne sont pas acteurs jouent la comédie. Dans une interview, Lepage explique que Michel arbore le masque d'un révolutionnaire qui rêve de faire son entrée sur scène (Coulombe 100). 
${ }^{14}$ Notons que la comparaison qu'ébauche Lepage entre son film et le théâtre nô ne se cantonne pas uniquement aux recoupements thématiques. Par de nombreuses rimes visuelles, Lepage s'emploie à raffermir le rapport entre son film et le théâtre qui lui donne son nom, ainsi qu'au film de Resnais. Un exemple parmi d'autres: à l'image de la Française d'Hiroshima mon amour qui se passe la tête sous l'eau avant de réciter sa litanie de l'oubli devant le miroir, répondent les plans de Sophie quittant le spectacle nô pour aller s'asperger le visage d'eau froide dans la salle de toilettes, pendant que l'acteur nô masqué mime ce même geste sur la scène.

${ }^{15}$ Dans Les Sept branches, Patricia remarque qu'“[à] Paris, il donnait dans l'avant-garde, et maintenant il essaie de se faire une carrière dans le vaudeville à Montréal” (62).

${ }^{16}$ Sophie tient le rôle de la Môme Crevette, danseuse du Moulin Rouge et maîtresse de Petypon, lequel achète ses faveurs tout en se moquant de son parler populaire. Toujours est-il que lorsque l'oncle respectable et fortuné de Petypon prend la Môme Crevette pour l'épouse légitime de son neveu, celui-ci la supplie de se faire passer pour une dame parisienne. Elle donne si bien le change que les bourgeoises provinciales s'empressent d'imiter sa manière de s'exprimer. 